\title{
INVESTIGATION OF SPORTS-SPECIFIC PSYCHOLOGICAL ABILITY LEVELS OF HIGH SCHOOL STUDENTS
}

\author{
Kemal Kurak ${ }^{1}$, \\ Hakan Büyükçelebi ${ }^{2 i}$ \\ 'Department of Coaching Education, \\ Çanakkale Onsekiz Mart University, \\ Turkey \\ ${ }^{2}$ Department of Coaching Education, \\ Inonu University, \\ Turkey
}

\begin{abstract}
:
The aim of this study is to investigate the sports-specific psychological ability levels of high school students. The study is a descriptive study, and the data were collected by survey method. A total of 402 students (226 male, 176 female) participated in the study. Mann Whitney $\mathrm{U}$ test was used for paired comparisons and Kruscal Wallis H tests for multiple comparisons. The Mann Whitney $U$ test used for non-parametric data was utilized to detect the source of the difference in multiple comparisons. In the study, the significance level was accepted as $\mathrm{p}<0.05$. There was a statistically significant difference in favor of male students in the sub-dimension of goal setting, in all sub-dimensions except for the sub-dimension of being open to learning according to high school type variable, in favor of those who define themselves social-extroverted in all sub-dimensions except for high performance under pressure according to the self-definition variable, in favor of those who do team sport in the sub-dimensions of goal setting, confidence and achievement motive according to the sport branch variable; and in all sub-dimensions of the variables of club license status, sport year, number of exercise per week, being in the school team and presence of a licensed athlete in the family. As a result of this study, it was found that students who were successful in bilateral relations, social and were interested in sports for a long time were more advanced in terms of sports-specific psychological ability.
\end{abstract}

Keywords: education, student, sports, high school, stress

i Correspondence: email hakan.buyukcelebi@inonu.edu.tr 


\section{Introduction}

With the increase of expectations from athletes, whereas all kinds of factors that can affect sports success are examined extensively, the opinion that the psychological characteristics are as effective as physical characteristics in the success of athletes has gained more importance day by day (Malina et al., 2007).

The factors affecting the final result of sporting competitions play an important role in the mixed structure of sporting performance. In general, psychological factors are the leading factors that affect performance positively and negatively. The athlete's subconscious awareness of the current gains after a long period of work in line with his / her existing abilities and skills and the motivation to be shaped depending on these gains can neutralize psychological factors (Aktaş, 2009).

Some athletes with the same physical characteristics and training background might be considered mentally superior to their peers. Some athletes can display better performance when they are under pressure, use their game plans more effectively, find more creative solutions against challenges and resist, concentrate more intensively, push their limits more, learn new techniques more quickly or prepare better for competitions compared to their physical counterparts. Mental advantages of the athlete are evaluated within the science of sports psychology. While the science of psychology is defined as the science that tries to explain behaviors and mental processes, Sports Psychology is defined as a science that tries to explain mental processes in a sportive context.

When children and young athletes are evaluated in terms of the results of sports psychology considering the various social environments in which they exist, their coaches, teachers, family members and peers are stated to have different impacts on athletes (McCarthy, Jones, Harwood and Olivier, 2010). According to Gee, Marshall and King (2010), Sportive Psychological Ability refers to the combination of characteristics that must be possessed by children and adolescents to display high performance; the absence of one or more of these characteristics might adversely affect the performance. Harwood, Cumming, and Fletcher emphasize that young athletes with a high-quality Sportive Psychological Ability need to follow a holistic approach in terms of Psychological Ability to improve performance especially in sporting competitions carried out under high pressure (Harwood, Cumming and Fletcher, 2004). Determination of psychological ability from a sports point of view is introduced as an important factor in specifying in which direction the performance can be (Hayslip, Petrie, MacIntire and Jones, 2010), it is also stated that a positive contribution can be made to performance improvement through the psychological characteristics such as mental preparation, coping with anxiety, confidence, concentration and motivation as well as the techniques such as goal setting, relaxation, visualization and self-talk (Weinberg, Miller and Horn, 2012).

Anxiety; anxiety is a state of uneasiness that occurs when a desire appears to be unreachable (Cevizci, 1999). Therefore, anxiety is a distressing emotion arising from insecurity or lack of confidence (Bakırcıoğlu, 2012). In this regard, anxiety is a series of 
negative emotional states associated with the inability to cope with the stress caused by environmental factors (Hagger, 2005).

Sport requires attention, knowledge and attention. For this reason, optimal anxiety is necessary for the sport person to achieve the desired performance and success. The high level of anxiety is expressed as "start rush" while the low level of anxiety is expressed as "start laziness" (Tavacioğlu, 1999). At this point, increasing anxiety in sporting activities will affect the performance negatively and this might cause emotional deficiencies such as psychological destruction and low self-confidence.

Scientists who steer sport are in a great effort to take the performance to the top. They develop new training models and search for the ways to get higher levels of efficiency from the athlete. All these scientific studies have shown that not only physical capacity is decisive in sport performance, but psychological factors are a quite important parameter in sporting performance (Akarçeşme, 2004). Performance is a process formed by the combination of physical and psychological parameters. The athlete might suffer both economic and professional losses as a result of failure due to performance impairment. This causes the athlete to get under high pressure before the competition and fails in the competition upon not showing his/her real performance (Akarçeşme, 2004). Considering that most elite athletes have very good skills in psychological capacities such as motivation, anxiety management, coping with competition stress, concentration and goal setting alongside their physical and physiological capacities, it should be known that the psychological dimension should not be ignored in enhancing sportive performance (Koç, 2004).

A physical capacity at the highest level is not enough for a solid sports performance. Psychological capacity is essential for an excellent performance. Today, modern researches draw attention to an important point and underlines the importance of mental factors along with motoric features. At this point, when the studies in this field conducted are examined, it is seen that there are not enough researches about mental factors and this deficiency is more in our country (Efe et al., 2008).

\section{Method}

\subsection{Population and Sample / Research Group}

The population of the research consists of individuals who receive education in high schools in the province of Mersin and its districts in 2019-2020 academic year. The sample group comprises a total of 402 students (226 male, 176 female) from Anatolian high school, vocational high school, sports high school and science high school.

\subsection{Data Collection}

The data obtained from the research was collected through the personal information form prepared by the researcher and "The Athletic Coping Skills Inventory (ACSI-28)" which was developed by Smith, Schutz, Smoll and Ptacek (1995) and adapted to Turkish by Özcan and Günay (2017) as "(Coping Skills Inventory for Sportive Problems) Sportif Sorunlarla Başa Çıkma Becerileri Envanteri". 


\subsection{Personal Information Form}

In order to reveal the information about the personal characteristics of the research group and to determine how they are shaped according to the characteristics (independent variables) that affect their coping skills with sport problems, the information form including questions related to age, gender, number of exercises per week, type of school, purpose of sport, type of play liked, how they define themselves, education level of the mother and father, branch of sports, the status of playing in the school team, whether there are licensed athletes in the family, sport year and club license status were used.

\subsection{Athletic Coping Skills Inventory (ACSI-28)}

The scale developed by Smith et al. (1995) aimed to measure athletes' psychological coping ability with difficulties. The structure of the ACSI-28 Scale consists of 28 items and 7 sub-factors. In the scale developed in the form of 4-point Likert-type, the participants were asked to indicate how often they had experiences (almost never $=0$, sometimes $=1$, often $=2$, almost always $=3$ ). The total score interval for each subscale is indicated as the lowest 0 and the highest 12 , and the higher score obtained from the subscale is expressed as a stronger indicator.

\subsection{Data Analysis}

Statistical package program (SPSS 22 Version) was used to analyze the data to be obtained from the research. In order to understand which tests should be applied before statistical analysis, it was tested whether the data was normally distributed or not. The Mann Whitney U test was used for paired comparisons and Kruscal Wallis $\mathrm{H}$ tests for multiple comparisons. The Mann Whitney $U$ test used for non-parametric data was utilized to detect the source of the difference in multiple comparisons. In the study, significance level was accepted as $\mathrm{p}<0.05$.

\section{Results}

Table 1: Frequency values of the research group

\begin{tabular}{|c|c|c|}
\hline & $\mathbf{N}$ & $\%$ \\
\hline \multicolumn{3}{|l|}{ Age } \\
\hline 15 & 95 & 23,6 \\
\hline 16 & 120 & 29,9 \\
\hline 17 & 123 & 30,6 \\
\hline 18 & 64 & 15,9 \\
\hline Total & 402 & 100 \\
\hline \multicolumn{3}{|l|}{ Gender } \\
\hline Male & 226 & 56,2 \\
\hline Female & 176 & 43,8 \\
\hline Total & 402 & 100 \\
\hline \multicolumn{3}{|c|}{ High School Type } \\
\hline Anatolian & 134 & 33,3 \\
\hline Vocational & 72 & 17,9 \\
\hline Sports & 112 & 27,9 \\
\hline
\end{tabular}


INVESTIGATION OF SPORTS-SPECIFIC PSYCHOLOGICAL ABILITY LEVELS OF HIGH SCHOOL STUDENTS

\begin{tabular}{|l|c|c|}
\hline \hline Science & 84 & 20,9 \\
\hline Total & 402 & 100 \\
\hline Sport Year & 124 & 30,8 \\
\hline $0-2$ years & 116 & 28,9 \\
\hline 3-5 years & 76 & 18,9 \\
\hline 6-7 years & 86 & 21,4 \\
\hline 8 years and more & 402 & 100 \\
\hline Total & \multicolumn{3}{l|}{} \\
\hline Weekly Training/Exercise Number & 67 & 16,7 \\
\hline 1 & 80 & 19,9 \\
\hline 2 & 68 & 16,9 \\
\hline 3 & 96 & 23,9 \\
\hline 4 & 91 & 22,6 \\
\hline 5 and more & 402 & 100 \\
\hline Total & \multicolumn{2}{l|}{} \\
\hline Branch & 168 & 41,8 \\
\hline Individual Sport & 234 & 58,2 \\
\hline Team Sport & 402 & 100 \\
\hline Total & \multicolumn{2}{l|}{} \\
\hline Club License & 207 & 51,5 \\
\hline Yes & 195 & 48,5 \\
\hline No & 402 & 100 \\
\hline Total & \multicolumn{2}{l|}{} \\
\hline
\end{tabular}

Table 2: Frequency values of the research group

\begin{tabular}{|c|c|c|}
\hline & $\mathbf{N}$ & $\%$ \\
\hline \multicolumn{3}{|l|}{ Mother's Education Level } \\
\hline Primary School & 176 & 43,8 \\
\hline Secondary School & 91 & 22,6 \\
\hline High School & 63 & 15,7 \\
\hline University & 72 & 17,9 \\
\hline Total & 402 & 100 \\
\hline \multicolumn{3}{|l|}{ Play Type Liked } \\
\hline Associative Play & 98 & 24,4 \\
\hline Cooperative Play & 230 & 57,2 \\
\hline Solitary (Independent) Play & 74 & 18,4 \\
\hline Total & 402 & 100 \\
\hline \multicolumn{3}{|l|}{ Father's Education Level } \\
\hline Primary School & 106 & 26,4 \\
\hline Secondary School & 119 & 29,6 \\
\hline High School & 93 & 23,1 \\
\hline University & 84 & 20,9 \\
\hline Total & 402 & 100 \\
\hline \multicolumn{3}{|l|}{ Purpose of Sport } \\
\hline Winning & 124 & 30,8 \\
\hline Improvement & 116 & 28,9 \\
\hline Entertainment, Enjoyment & 162 & 40,3 \\
\hline Total & 402 & 100 \\
\hline
\end{tabular}




\begin{tabular}{|l|c|c|}
\hline \hline Playing in the School Team & 201 & 49,6 \\
\hline Yes & 204 & 50,4 \\
\hline No & 405 & 100 \\
\hline Total & 137 & 34,1 \\
\hline Licensed Athlete in the Family & 265 & 65,9 \\
\hline Yes & 402 & 100 \\
\hline No & & \\
\hline Total & 183 & 45,5 \\
\hline Self-Definition & 219 & 54,5 \\
\hline Asocial-Introvert & 402 & 100 \\
\hline Social-Extrovert &
\end{tabular}

Table 3: Analysis results of the research group by the gender variable

\begin{tabular}{|c|c|c|c|c|c|c|}
\hline Sub-dimensions & Gender & $\mathbf{N}$ & $\bar{x}$ & Ss & $\mathbf{t}$ & $\mathbf{p}$ \\
\hline \multirow{2}{*}{$\begin{array}{l}\text { Goal Setting/ } \\
\text { Mental Preparation }\end{array}$} & Male & 226 & 1,79 & ,66 & \multirow{2}{*}{$-2,504$} & \multirow{2}{*}{, $012^{*}$} \\
\hline & Female & 176 & 1,62 & ,74 & & \\
\hline \multirow{2}{*}{ Concentration } & Male & 226 & 1,93 & ,61 & \multirow{2}{*}{$-2,529$} & \multirow{2}{*}{, $011^{*}$} \\
\hline & Female & 176 & 1,76 & ,66 & & \\
\hline \multirow{2}{*}{ Free from Anxiety } & Male & 226 & 1,84 & ,78 & \multirow{2}{*}{,- 297} & \multirow{2}{*}{,767 } \\
\hline & Female & 176 & 1,85 & 83 & & \\
\hline \multirow{2}{*}{$\begin{array}{l}\text { High Performance } \\
\text { under Pressure }\end{array}$} & Male & 226 & 1,36 & ,73 & \multirow{2}{*}{$-5,359$} & \multirow{2}{*}{, $000^{*}$} \\
\hline & Female & 176 & ,98 & ,72 & & \\
\hline \multirow{2}{*}{ Coping with Difficulty } & Male & 226 & 1,85 & 70 & \multirow{2}{*}{$-2,314$} & \multirow{2}{*}{, $021^{*}$} \\
\hline & Female & 176 & 1,69 & ,75 & & \\
\hline \multirow{2}{*}{\begin{tabular}{|l} 
Confidence and \\
Achievement Motive
\end{tabular}} & Male & 226 & 2,19 & ,64 & \multirow{2}{*}{$-1,265$} & \multirow{2}{*}{,206 } \\
\hline & Female & 176 & 2,08 & ,73 & & \\
\hline \multirow{2}{*}{ Coaching Status } & Male & 226 & 2,26 & ,57 & \multirow{2}{*}{$-1,462$} & \multirow{2}{*}{,144 } \\
\hline & Female & 176 & 2,15 & ,66 & & \\
\hline
\end{tabular}

According to Table 3, it was determined that there was a statistically significant difference in favor of male students in terms of goal setting / mental preparation, concentration, high performance under pressure and coping with difficulty according to the gender variable of the research group.

Table 4: Analysis results of the research group by the age variable

\begin{tabular}{|c|c|c|c|c|c|c|c|}
\hline Sub-dimensions & Age & $\mathbf{N}$ & $\bar{x}$ & SD & $\mathbf{X}^{2}$ & $\mathbf{p}$ & Difference U Test \\
\hline \multirow{4}{*}{$\begin{array}{l}\text { Goal Setting/ } \\
\text { Mental Preparation }\end{array}$} & a-15 & 95 & 1,66 & ,68 & \multirow{4}{*}{2,770} & \multirow{4}{*}{,429 } & \\
\hline & b-16 & 120 & 1,67 & ,73 & & & \\
\hline & $\mathrm{c}-17$ & 123 & 1,77 & ,72 & & & \\
\hline & d-18 & 64 & 1,76 & ,62 & & & \\
\hline \multirow{4}{*}{ Concentration } & a-15 & 95 & 1,81 & ,70 & \multirow{4}{*}{,402 } & \multirow{4}{*}{,940 } & \\
\hline & b-16 & 120 & 1,87 & 64 & & & \\
\hline & c-17 & 123 & 1,86 & ,62 & & & \\
\hline & d-18 & 64 & 1,89 & ,58 & & & \\
\hline \multirow{3}{*}{$\begin{array}{l}\text { Free from } \\
\text { Anxiety }\end{array}$} & a-15 & 95 & 1,74 & 88 & \multirow{3}{*}{1,555} & \multirow{3}{*}{670} & \\
\hline & b-16 & 120 & 1,88 & 84 & & & \\
\hline & c-17 & 123 & 1,87 & 69 & & & \\
\hline
\end{tabular}




\begin{tabular}{|c|c|c|c|c|c|c|c|}
\hline & d-18 & 64 & 1,88 & 83 & & & \\
\hline \multirow{4}{*}{$\begin{array}{l}\text { High Performance } \\
\text { under Pressure }\end{array}$} & a-15 & 95 & 1,09 & ,68 & \multirow{4}{*}{2,284} & \multirow{4}{*}{,516 } & \\
\hline & b-16 & 120 & 1,23 & ,75 & & & \\
\hline & c-17 & 123 & 122 & ,75 & & & \\
\hline & d-18 & 64 & 1,23 & , 85 & & & \\
\hline \multirow{4}{*}{$\begin{array}{l}\text { Coping } \\
\text { with Difficulty }\end{array}$} & a-15 & 95 & 1,70 & ,76 & \multirow{4}{*}{1,278} & \multirow{4}{*}{ 734 } & \\
\hline & b-16 & 120 & 1,80 & ,78 & & & \\
\hline & c-17 & 123 & 1,81 & ,67 & & & \\
\hline & d-18 & 64 & 1,82 & ,71 & & & \\
\hline \multirow{4}{*}{$\begin{array}{l}\text { Confidence and } \\
\text { Achievement Motive }\end{array}$} & a-15 & 95 & 2,11 & ,70 & \multirow{4}{*}{870} & \multirow{4}{*}{ 833 } & \\
\hline & b-16 & 120 & 2,12 & ,70 & & & \\
\hline & c-17 & 123 & 2,19 & ,66 & & & \\
\hline & d-18 & 64 & 2,12 & ,68 & & & \\
\hline \multirow{4}{*}{$\begin{array}{l}\text { Coaching } \\
\text { Status }\end{array}$} & a-15 & 95 & 2,25 & ,59 & \multirow{4}{*}{6,478} & \multirow{4}{*}{ 091 } & \\
\hline & b-16 & 120 & 2,28 & ,65 & & & \\
\hline & c-17 & 123 & 2,20 & ,57 & & & \\
\hline & d-18 & 64 & 2,05 & 66 & & & \\
\hline
\end{tabular}

According to Table 4, no statistically significant difference was observed in any subdimension related to the skills of the research group to deal with sporting problems depending on the age variable.

Table 5: Analysis results of the study group by high school type variable

\begin{tabular}{|c|c|c|c|c|c|c|c|}
\hline Sub-dimensions & High School Type & $\mathbf{N}$ & $\bar{x}$ & SD & $\mathbf{X}^{2}$ & $p$ & Difference U Test \\
\hline \multirow{4}{*}{$\begin{array}{l}\text { Goal Setting/ } \\
\text { Mental Preparation }\end{array}$} & a. Anatolian & 134 & 1,76 & 64 & \multirow{4}{*}{26,233} & \multirow{4}{*}{, $000^{*}$} & \multirow{4}{*}{$\begin{array}{c}c>a, b, d \\
a>d\end{array}$} \\
\hline & b. Vocational & 72 & 1,62 & 82 & & & \\
\hline & c. Sports & 112 & 1,93 & 62 & & & \\
\hline & d. Science & 84 & 1,42 & 67 & & & \\
\hline \multirow{4}{*}{ Concentration } & a. Anatolian & 134 & 1,86 & 61 & \multirow{4}{*}{11,066} & \multirow{4}{*}{, $011^{*}$} & \multirow{4}{*}{$\mathrm{c}>\mathrm{d}$} \\
\hline & b. Vocational & 72 & 1,82 & ,71 & & & \\
\hline & c. Sports & 112 & 2,00 & 62 & & & \\
\hline & d. Science & 84 & 1,69 & 60 & & & \\
\hline \multirow{4}{*}{$\begin{array}{l}\text { Free } \\
\text { from Anxiety }\end{array}$} & a. Anatolian & 134 & 1,95 & ,73 & \multirow{4}{*}{13,379} & \multirow{4}{*}{,004 } & \multirow{4}{*}{$a, b>c, d$} \\
\hline & b. Vocational & 72 & 2,02 & 86 & & & \\
\hline & c. Sports & 112 & 1,71 & 79 & & & \\
\hline & d. Science & 84 & 1,71 & 84 & & & \\
\hline \multirow{4}{*}{$\begin{array}{l}\text { High Performance } \\
\text { under Pressure }\end{array}$} & a. Anatolian & 134 & 1,30 & ,73 & \multirow{4}{*}{21,234} & \multirow{4}{*}{, $000^{*}$} & \multirow{4}{*}{$a, c>b, d$} \\
\hline & b. Vocational & 72 & ,95 & ,70 & & & \\
\hline & c. Sports & 112 & 1,37 & ,76 & & & \\
\hline & d. Science & 84 & 1,02 & ,73 & & & \\
\hline \multirow{4}{*}{$\begin{array}{l}\text { Coping } \\
\text { with Difficulty }\end{array}$} & a. Anatolian & 134 & 1,79 & 72 & \multirow{4}{*}{9,291} & \multirow{4}{*}{, $026^{*}$} & \multirow{4}{*}{$c>d$} \\
\hline & b. Vocational & 72 & 1,76 & 80 & & & \\
\hline & c. Sports & 112 & 1,92 & ,72 & & & \\
\hline & d. Science & 84 & 1,60 & ,66 & & & \\
\hline \multirow{4}{*}{$\begin{array}{l}\text { Confidence and } \\
\text { Achievement Motive }\end{array}$} & a. Anatolian & 134 & 2,14 & 65 & \multirow{4}{*}{10,832} & \multirow{4}{*}{, $013^{*}$} & \multirow{4}{*}{$c>d$} \\
\hline & b. Vocational & 72 & 2,05 & 83 & & & \\
\hline & c. Sports & 112 & 2,30 & 60 & & & \\
\hline & d. Science & 84 & 1,99 & ,66 & & & \\
\hline
\end{tabular}




\begin{tabular}{|c|c|c|c|c|c|c|}
\hline \multirow{4}{*}{ Coaching Status } & a. Anatolian & 134 & 2,19 & ,65 & \multirow{4}{*}{3,620} & \multirow{3}{*}{,306 } \\
\hline & b. Vocational & 72 & 2,27 & ,70 & & \\
\hline & c. Sports & 112 & 2,24 &, 58 & & \\
\hline & d. Science & 84 & 2,15 &, 53 & & \\
\hline
\end{tabular}

According to Table 5, it was found that there was a statistically significant difference in all sub-dimensions except for the sub-dimension of being open to learning in the research group by the high school type variable $(\mathrm{p}<0.05)$.

Table 6: Analysis results of the research group by the mother's education level variable

\begin{tabular}{|c|c|c|c|c|c|c|c|}
\hline Sub-dimension & Education Level of Mother & $\mathbf{N}$ & $\bar{x}$ & SD & $\mathbf{X}^{2}$ & p & Difference U Test \\
\hline \multirow{4}{*}{$\begin{array}{l}\text { Goal Setting/ } \\
\text { Mental Preparation }\end{array}$} & a. Primary school & 176 & 1,77 & ,68 & \multirow{4}{*}{8,773} & \multirow{4}{*}{, $032^{*}$} & \multirow{4}{*}{$a, b>c, d$} \\
\hline & b. Secondary school & 91 & 1,81 & ,64 & & & \\
\hline & c. High School & 63 & 1,54 & ,71 & & & \\
\hline & d. University & 72 & 1,59 &, 76 & & & \\
\hline \multirow{4}{*}{ Concentration } & a. Primary School & 176 & 1,89 & ,64 & \multirow{4}{*}{1,404} & \multirow{4}{*}{,705 } & \\
\hline & b. Secondary School & 91 & 1,84 & ,64 & & & \\
\hline & c. High School & 63 & 1,78 & ,63 & & & \\
\hline & d. University & 72 & 1,87 & ,63 & & & \\
\hline \multirow{4}{*}{$\begin{array}{l}\text { Free } \\
\text { from Anxiety }\end{array}$} & a. Primary school & 176 & 1,86 & ,77 & \multirow{4}{*}{1,985} & \multirow{4}{*}{,575 } & \\
\hline & b. Secondary School & 91 & 1,91 & ,83 & & & \\
\hline & c. High School & 63 & 1,74 & ,82 & & & \\
\hline & d. University & 72 & 1,81 & ,85 & & & \\
\hline \multirow{4}{*}{$\begin{array}{l}\text { High Performance } \\
\text { under Pressure }\end{array}$} & a. Primary school & 176 & 1,24 & ,72 & \multirow{4}{*}{2,647} & \multirow{4}{*}{, 449 } & \\
\hline & b. Secondary School & 91 & 1,23 & ,78 & & & \\
\hline & c. High School & 63 & 1,11 & ,66 & & & \\
\hline & d. University & 72 & 1,12 & ,85 & & & \\
\hline \multirow{4}{*}{$\begin{array}{l}\text { Coping } \\
\text { with Difficulty }\end{array}$} & a. Primary School & 176 & 1,80 &, 72 & \multirow{4}{*}{,544 } & \multirow{4}{*}{,909 } & \\
\hline & b. Secondary school & 91 & 1,80 & ,73 & & & \\
\hline & \begin{tabular}{|l} 
c. High school \\
\end{tabular} & 63 & 1,73 & ,78 & & & \\
\hline & d. University & 72 & 1,75 & ,71 & & & \\
\hline \multirow{4}{*}{$\begin{array}{l}\text { Confidence and } \\
\text { Achievement Motive }\end{array}$} & a. Primary School & 176 & 2,21 & ,66 & \multirow{4}{*}{3,553} & \multirow{4}{*}{,314 } & \\
\hline & b. Secondary school & 91 & 2,13 & ,69 & & & \\
\hline & c. High school & 63 & 2,06 & ,73 & & & \\
\hline & d. University & 72 & 2,06 & ,70 & & & \\
\hline \multirow{4}{*}{ Coaching Status } & a. Primary school & 176 & 2,26 & ,60 & \multirow{4}{*}{2,574} & \multirow{4}{*}{462} & \\
\hline & b. Secondary school & 91 & 2,13 & ,67 & & & \\
\hline & c. High school & 63 & 2,15 & ,65 & & & \\
\hline & d. University & 72 & 2,26 &, 54 & & & \\
\hline
\end{tabular}

According to Table 6, a statistically significant difference was found only in the Goal Setting / Mental Preparation sub-dimension according to the mother's education level variable of the research group $(\mathrm{p}<0.05)$. 
Table 7: Analysis results of the research group by the father's education level variable

\begin{tabular}{|c|c|c|c|c|c|c|c|}
\hline Sub-dimension & Education Level of Father & $\mathbf{N}$ & $\bar{x}$ & SD & $\mathbf{X}^{2}$ & p & Difference U Test \\
\hline \multirow{4}{*}{$\begin{array}{l}\text { Goal Setting/ } \\
\text { Mental Preparation }\end{array}$} & a. Primary school & 106 & 1,81 & 62 & \multirow{4}{*}{11,893} & \multirow{4}{*}{$3,008^{*}$} & \multirow{4}{*}{$d<a, b, c$} \\
\hline & b. Secondary school & 119 & 1,70 & 69 & & & \\
\hline & c. High school & 93 & 1,82 & 72 & & & \\
\hline & d. University & 84 & 1,49 & 73 & & & \\
\hline \multirow{4}{*}{ Concentration } & a. Primary school & 106 & 1,83 &, 59 & \multirow{4}{*}{4,158} & \multirow{4}{*}{,245 } & \\
\hline & b. Secondary school & 119 & 1,86 & 71 & & & \\
\hline & c. High school & 93 & 1,96 & 60 & & & \\
\hline & d. University & 84 & 1,77 & 61 & & & \\
\hline \multirow{4}{*}{$\begin{array}{l}\text { Free } \\
\text { from Anxiety }\end{array}$} & a. Primary school & 106 & 1,77 & 77 & \multirow{4}{*}{3,609} & \multirow{4}{*}{,307 } & \\
\hline & b. Secondary school & 119 & 1,89 & 82 & & & \\
\hline & c. High school & 93 & 1,92 & ,77 & & & \\
\hline & d. University & 84 & 1,78 & 86 & & & \\
\hline \multirow{4}{*}{$\begin{array}{l}\text { High Performance } \\
\text { under Pressure }\end{array}$} & a. Primary school & 106 & 1,24 & 69 & \multirow{4}{*}{4,481} & \multirow{4}{*}{,214 } & \\
\hline & b. Secondary school & 119 & 1,27 & ,78 & & & \\
\hline & c. High school & 93 & 1,17 & 75 & & & \\
\hline & d. University & 84 & 1,06 & ,77 & & & \\
\hline \multirow{4}{*}{$\begin{array}{l}\text { Coping } \\
\text { with Difficulty }\end{array}$} & a. Primary school & 106 & 1,76 & 72 & \multirow{4}{*}{6,089} & \multirow{4}{*}{, 107} & \\
\hline & b. Secondary school & 119 & 1,88 &, 77 & & & \\
\hline & c. High school & 93 & 1,83 & 71 & & & \\
\hline & d. University & 84 & 1,62 & 69 & & & \\
\hline \multirow{4}{*}{$\begin{array}{l}\text { Confidence and } \\
\text { Achievement Motive }\end{array}$} & a. Primary school & 106 & 2,21 & 65 & \multirow{4}{*}{4,521} & \multirow{4}{*}{,210 } & \\
\hline & b. Secondary school & 119 & 2,16 &, 74 & & & \\
\hline & c. High school & 93 & 2,15 & 64 & & & \\
\hline & d. University & 84 & 2,01 & 68 & & & \\
\hline \multirow{4}{*}{ Coaching Status } & a. Primary school & 106 & 2,20 & 64 & \multirow{4}{*}{1,186} & \multirow{4}{*}{,756 } & \\
\hline & b. Secondary school & 119 & 2,25 & 61 & & & \\
\hline & c. High school & 93 & 2,20 & 64 & & & \\
\hline & d. University & 84 & 2,18 &, 57 & & & \\
\hline
\end{tabular}

Table 7 points out a statistically significant difference in the Goal Setting / Mental Preparation sub-dimension of the research group according to the variable of father's education level $(\mathrm{p}<0.05)$.

Table 8: Analysis results of the research group by the variable of Play Type Liked

\begin{tabular}{|c|c|c|c|c|c|c|c|}
\hline Sub-dimensions & Play Type Liked & $\mathbf{N}$ & $\bar{x}$ & SD & $\mathbf{X}^{2}$ & $\mathrm{p}$ & Difference U Test \\
\hline \multirow{3}{*}{$\begin{array}{l}\text { Goal Setting/ } \\
\text { Mental Preparation }\end{array}$} & a. Associative & 98 & 1,68 & ,71 & \multirow{3}{*}{ 234 } & \multirow{3}{*}{,889 } & \\
\hline & b. Cooperative & 230 & 1,72 & ,66 & & & \\
\hline & c. Solitary (Independent) & 74 & 1,73 & ,79 & & & \\
\hline \multirow{3}{*}{ Concentration } & a. Associative & 98 & 1,87 &, 63 & \multirow{3}{*}{1,663} & \multirow{3}{*}{,435 } & \\
\hline & b. Cooperative & 230 & 1,88 & ,64 & & & \\
\hline & c. Solitary (Independent) & 74 & 1,76 & ,65 & & & \\
\hline \multirow{3}{*}{$\begin{array}{l}\text { Free from } \\
\text { Anxiety }\end{array}$} & a. Associative & 98 & 1,93 & ,78 & \multirow{3}{*}{4,088} & \multirow{3}{*}{,129 } & \\
\hline & b. Cooperative & 230 & 1,85 &, 80 & & & \\
\hline & c. Solitary (Independent) & 74 & 1,70 & ,83 & & & \\
\hline \multirow{2}{*}{$\begin{array}{l}\text { High Performance } \\
\text { under Pressure }\end{array}$} & a. Associative & 98 & 1,18 &, 75 & \multirow{2}{*}{ 331 } & \multirow{2}{*}{847} & \\
\hline & b. Cooperative & 230 & 1,18 & ,75 & & & \\
\hline
\end{tabular}




\begin{tabular}{|c|c|c|c|c|c|c|c|}
\hline & c. Solitary (Independent) & 74 & 1,25 & 77 & & & \\
\hline \multirow{3}{*}{$\begin{array}{l}\text { Coping } \\
\text { with Difficulty }\end{array}$} & a. Associative & 98 & 1,89 & 73 & \multirow{3}{*}{8,164} & \multirow{3}{*}{, $017^{*}$} & \multirow{3}{*}{$c<a, b$} \\
\hline & b. Cooperative & 230 & 1,80 & 73 & & & \\
\hline & c. Solitary (Independent) & 74 & 1,58 & 70 & & & \\
\hline \multirow{3}{*}{$\begin{array}{l}\text { Confidence and } \\
\text { Achievement Motive }\end{array}$} & a. Associative & 98 & 2,18 & 65 & \multirow{3}{*}{4,535} & \multirow{3}{*}{104} & \\
\hline & b. Cooperative & 230 & 2,18 & 65 & & & \\
\hline & c. Solitary (Independent) & 74 & 1,95 & 80 & & & \\
\hline \multirow{3}{*}{ Coaching Status } & a. Associative & 98 & 2,20 &, 66 & \multirow{3}{*}{2,378} & \multirow{3}{*}{,304 } & \\
\hline & b. Cooperative & 230 & 2,25 &, 59 & & & \\
\hline & c. Solitary (Independent) & 74 & 2,12 & 63 & & & \\
\hline
\end{tabular}

Table 8 indicates a statistically significant difference only in the sub-dimension of coping with difficulty according to the variable of play type liked of the research group $(p<0.05)$.

Table 9: Analysis results of the research group by the Sport Purpose variable

\begin{tabular}{|c|c|c|c|c|c|c|c|}
\hline Sub-dimensions & Sport Purpose & $\mathbf{N}$ & $\bar{x}$ & SD & $X^{2}$ & $\mathrm{p}$ & Difference U Test \\
\hline \multirow{3}{*}{$\begin{array}{l}\text { Goal Setting/ } \\
\text { Mental Preparation }\end{array}$} & a. Winning & 124 & 1,73 & ,71 & \multirow{3}{*}{7,007} & \multirow{3}{*}{, $030^{*}$} & \multirow{3}{*}{$b>c$} \\
\hline & b. Improvement & 116 & 1,85 & ,60 & & & \\
\hline & c. Entertainment & 162 & 1,60 & ,73 & & & \\
\hline \multirow{3}{*}{ Concentration } & a. Winning & 124 & 1,97 & ,69 & \multirow{3}{*}{12,593} & \multirow{3}{*}{, $002^{*}$} & \multirow{3}{*}{$b>c$} \\
\hline & b. Improvement & 116 & 1,91 &, 59 & & & \\
\hline & c. Entertainment & 162 & 1,73 & ,61 & & & \\
\hline \multirow{3}{*}{ Free from Anxiety } & a. Winning & 124 & 1,87 & ,79 & \multirow{3}{*}{,126 } & \multirow{3}{*}{,939 } & \\
\hline & b. Improvement & 116 & 1,85 & ,75 & & & \\
\hline & c. Entertainment & 162 & 1,82 & ,85 & & & \\
\hline \multirow{3}{*}{$\begin{array}{l}\text { High Performance } \\
\text { under Pressure }\end{array}$} & a. Winning & 124 & 1,27 & ,81 & \multirow{3}{*}{6,862} & \multirow{3}{*}{, $032^{*}$} & \multirow{3}{*}{$b>c$} \\
\hline & b. Improvement & 116 & 1,29 & ,71 & & & \\
\hline & c. Entertainment & 162 & 1,08 & ,72 & & & \\
\hline \multirow{3}{*}{$\begin{array}{l}\text { Coping } \\
\text { with Difficulty }\end{array}$} & a. Winning & 124 & 1,71 &, 79 & \multirow{3}{*}{6,677} & \multirow{3}{*}{, $035^{*}$} & \multirow{3}{*}{$\begin{array}{c}b>a, c \\
a>c\end{array}$} \\
\hline & b. Improvement & 116 & 1,94 & ,67 & & & \\
\hline & c. Entertainment & 162 & 1,72 & ,71 & & & \\
\hline \multirow{3}{*}{$\begin{array}{l}\text { Confidence and } \\
\text { Achievement Motive }\end{array}$} & a. Winning & 124 & 2,15 & ,75 & \multirow{3}{*}{3,858} & \multirow{3}{*}{, 145 } & \\
\hline & b. Improvement & 116 & 2,22 & ,64 & & & \\
\hline & c. Entertainment & 162 & 2,07 & ,66 & & & \\
\hline \multirow{3}{*}{ Coaching Status } & a. Winning & 124 & 2,14 & ,65 & \multirow{3}{*}{3,408} & \multirow{3}{*}{, 182 } & \\
\hline & b. Improvement & 116 & 2,29 & ,60 & & & \\
\hline & c. Entertainment & 162 & 2,21 & ,60 & & & \\
\hline
\end{tabular}

Table 9 presents a statistically significant difference in the sub-dimensions of Goal setting / Mental preparation, Concentration, High performance under pressure and Coping with difficulty according to the Sport purpose variable of the research group $(p<0.05)$. 
Table 10: Analysis results of the research group by the Sport year variable

\begin{tabular}{|c|c|c|c|c|c|c|c|}
\hline Sub-dimensions & Sport Year & $\mathbf{N}$ & $\bar{x}$ & SD & $X^{2}$ & $\mathrm{p}$ & Difference U Test \\
\hline \multirow{4}{*}{$\begin{array}{l}\text { Goal Setting/ } \\
\text { Mental Preparation }\end{array}$} & a. $0-2$ yr. & 124 & 1,30 & ,59 & \multirow{4}{*}{85,106} & \multirow{4}{*}{, $000^{*}$} & \multirow{4}{*}{$a<b, c . d$} \\
\hline & b. 3-5 yr. & 116 & 1,73 & 66 & & & \\
\hline & c. $6-7 \mathrm{yr}$. & 76 & 1,88 & 65 & & & \\
\hline & d. 8 and + & 86 & 2,15 & 61 & & & \\
\hline \multirow{4}{*}{ Concentration } & a. $0-2 \mathrm{yr}$. & 124 & 1,44 &, 56 & \multirow{4}{*}{81,462} & \multirow{4}{*}{, $000^{*}$} & \multirow{4}{*}{$a<b, c . d$} \\
\hline & b. $3-5 \mathrm{yr}$. & 116 & 1,92 & ,63 & & & \\
\hline & c. $6-7 \mathrm{yr}$. & 76 & 2,12 &, 53 & & & \\
\hline & d. 8 and + & 86 & 2,13 &, 54 & & & \\
\hline \multirow{4}{*}{$\begin{array}{l}\text { Free from } \\
\text { Anxiety }\end{array}$} & a. $0-2 \mathrm{yr}$. & 124 & 1,53 & 81 & \multirow{4}{*}{26,625} & \multirow{4}{*}{, $000^{*}$} & \multirow{4}{*}{$a<b, c . d$} \\
\hline & b. 3-5 yr. & 116 & 1,96 & ,76 & & & \\
\hline & c. $6-7 \mathrm{yr}$. & 76 & 1,98 & ,75 & & & \\
\hline & d. 8 and + & 86 & 2,02 & ,77 & & & \\
\hline \multirow{4}{*}{$\begin{array}{l}\text { High Performance } \\
\text { under Pressure }\end{array}$} & a. $0-2 \mathrm{yr}$. & 124 & 1,97 & ,63 & \multirow{4}{*}{12,601} & \multirow{4}{*}{, $006^{*}$} & \multirow{4}{*}{$a<b, c . d$} \\
\hline & b. 3-5 yr. & 116 & 1,31 & ,76 & & & \\
\hline & c. $6-7 \mathrm{yr}$. & 76 & 1,31 & 83 & & & \\
\hline & d. 8 and + & 86 & 1,26 & ,76 & & & \\
\hline \multirow{4}{*}{$\begin{array}{l}\text { Coping } \\
\text { with Difficulty }\end{array}$} & a. $0-2 \mathrm{yr}$. & 124 & 1,40 & 67 & \multirow{4}{*}{59,153} & \multirow{4}{*}{, $000^{*}$} & \multirow{4}{*}{$a<b, c . d$} \\
\hline & b. 3-5 yr. & 116 & 1,81 & 67 & & & \\
\hline & c. $6-7 \mathrm{yr}$. & 76 & 1,96 & ,73 & & & \\
\hline & d. 8 and + & 86 & 2,14 & 64 & & & \\
\hline \multirow{4}{*}{$\begin{array}{l}\text { Confidence and } \\
\text { Achievement Motive }\end{array}$} & a. $0-2 \mathrm{yr}$. & 124 & 1,74 & ,71 & \multirow{4}{*}{63,885} & \multirow{4}{*}{, $000^{*}$} & \multirow{4}{*}{$a<b$, c.d } \\
\hline & b. 3-5 yr. & 116 & 2,22 &, 58 & & & \\
\hline & c. $6-7 \mathrm{yr}$. & 76 & 2,33 &, 57 & & & \\
\hline & d. 8 and + & 86 & 2,44 &, 60 & & & \\
\hline \multirow{4}{*}{$\begin{array}{l}\text { Coaching } \\
\text { Status }\end{array}$} & a. 0-2 yr. & 124 & 1,85 & ,65 & \multirow{4}{*}{58,993} & \multirow{4}{*}{, $000^{*}$} & \multirow{4}{*}{$a<b, c . d$} \\
\hline & b. 3-5 yr. & 116 & 2,32 &, 53 & & & \\
\hline & c. $6-7 \mathrm{yr}$. & 76 & 2,37 &, 52 & & & \\
\hline & d. 8 and + & 86 & 2,45 &, 52 & & & \\
\hline
\end{tabular}

According to Table 10, the difference was statistically significant in all sub-dimensions of coping skills with sport problems depending on the sport year variable of the research group ( $\mathrm{p}<0.05)$.

Table 11: Analysis results of the research group by the variable of being club-licensed

\begin{tabular}{|c|c|c|c|c|c|c|}
\hline Sub-dimensions & Club License & $\mathbf{N}$ & $\bar{x}$ & SD & $\mathrm{Z}$ & $\mathbf{P}$ \\
\hline \multirow{2}{*}{$\begin{array}{l}\text { Goal Setting/ } \\
\text { Mental Preparation }\end{array}$} & Yes & 207 & 1,94 & ,66 & \multirow{2}{*}{$-6,826$} & \multirow{2}{*}{, $000^{*}$} \\
\hline & $\mathrm{No}$ & 195 & 1,47 & 65 & & \\
\hline \multirow{2}{*}{ Concentration } & Yes & 207 & 2,07 &, 58 & \multirow{2}{*}{$-6,955$} & \multirow{2}{*}{, $000^{*}$} \\
\hline & No & 195 & 1,63 & ,61 & & \\
\hline \multirow{2}{*}{\begin{tabular}{|l|} 
Free from \\
Anxiety
\end{tabular}} & Yes & 207 & 1,96 & 80 & \multirow{2}{*}{$-2,895$} & \multirow{2}{*}{, $004^{*}$} \\
\hline & No & 195 & 1,73 & ,79 & & \\
\hline \multirow{2}{*}{$\begin{array}{l}\text { High Performance } \\
\text { under Pressure }\end{array}$} & Yes & 207 & 1,34 & ,78 & \multirow{2}{*}{$-3,481$} & \multirow{2}{*}{, $000^{*}$} \\
\hline & No & 195 & 1,05 & ,69 & & \\
\hline \multirow{2}{*}{$\begin{array}{l}\text { Coping } \\
\text { with Difficulty }\end{array}$} & Yes & 207 & 1,97 &, 70 & \multirow{2}{*}{$-5,422$} & \multirow{2}{*}{, $000^{*}$} \\
\hline & No & 195 & 1,58 & ,70 & & \\
\hline Confidence and & Yes & 207 & 2,34 & 60 & $-6,236$ &, $000^{*}$ \\
\hline
\end{tabular}


INVESTIGATION OF SPORTS-SPECIFIC PSYCHOLOGICAL ABILITY LEVELS OF HIGH SCHOOL STUDENTS

\begin{tabular}{|l|c|c|c|c|c|c|}
\hline \hline Achievement Motive & No & 195 & 1,92 &, 70 & & \\
\hline \multirow{2}{*}{ Coaching Status } & Yes & 207 & 2,35 &, 58 & \multirow{2}{*}{$-4,890$} & \multirow{2}{*}{, $000^{*}$} \\
\cline { 2 - 6 } & No & 195 & 2,06 &, 62 & \\
\hline
\end{tabular}

According to Table 11, it was found that the was statistically significant in favor of those who play in a sports club difference in all sub-dimensions of coping skills with sport problems depending on the club license variable of the research group $(p<0.05)$.

Table 12: Analysis results of the research group according to the variable of playing in the school team

\begin{tabular}{|c|c|c|c|c|c|c|}
\hline Sub-dimensions & School Team & $\mathbf{N}$ & $\bar{x}$ & SD & $\mathrm{Z}$ & $\mathbf{P}$ \\
\hline \multirow{2}{*}{\begin{tabular}{|l} 
Goal Setting/ \\
Mental Preparation
\end{tabular}} & Yes & 224 & 1,91 & 67 & \multirow{2}{*}{$-6,424$} & \multirow{2}{*}{, $000^{*}$} \\
\hline & No & 178 & 1,47 & 66 & & \\
\hline \multirow{2}{*}{ Concentration } & Yes & 224 & 2,00 & ,58 & \multirow{2}{*}{$-5,029$} & \multirow{2}{*}{, $000^{*}$} \\
\hline & No & 178 & 1,67 & 66 & & \\
\hline \multirow{2}{*}{$\begin{array}{l}\text { Free from } \\
\text { Anxiety }\end{array}$} & Yes & 224 & 2,00 &, 79 & \multirow{2}{*}{$-4,502$} & \multirow{2}{*}{, $000^{*}$} \\
\hline & No & 178 & 1,65 & ,78 & & \\
\hline \multirow{2}{*}{$\begin{array}{l}\text { High Performance } \\
\text { under Pressure }\end{array}$} & Yes & 224 & 1,33 &, 76 & \multirow{2}{*}{$-4,036$} & \multirow{2}{*}{, $000^{*}$} \\
\hline & No & 178 & 1,02 & ,70 & & \\
\hline \multirow{2}{*}{$\begin{array}{l}\text { Coping } \\
\text { with Difficulty }\end{array}$} & Yes & 224 & 1,96 & ,68 & \multirow{2}{*}{$-5,408$} & \multirow{2}{*}{, $000^{*}$} \\
\hline & No & 178 & 1,55 & ,72 & & \\
\hline \multirow{2}{*}{$\begin{array}{l}\text { Confidence and } \\
\text { Achievement Motive }\end{array}$} & Yes & 224 & 2,28 &, 59 & \multirow{2}{*}{$-4,064$} & \multirow{2}{*}{, $000^{*}$} \\
\hline & No & 178 & 1,97 & ,75 & & \\
\hline \multirow{2}{*}{$\begin{array}{l}\text { Coaching } \\
\text { Status }\end{array}$} & Yes & 224 & 2,34 &, 56 & \multirow{2}{*}{$-4,542$} & \multirow{2}{*}{, $000^{*}$} \\
\hline & No & 178 & 2,05 & ,64 & & \\
\hline
\end{tabular}

According to Table 12, the difference was statistically significant in favor of those who participate in sporting activities in school teams in all sub-dimensions of coping skills with sport problems depending on the variable of playing in the school team $(p<0.05)$.

Table 13: Analysis results of the research group according to the presence of licensed athletes in the family

\begin{tabular}{|c|c|c|c|c|c|c|}
\hline Sub-dimensions & Licensed Athlete in the Family & $\mathbf{N}$ & $\bar{x}$ & SD & $\mathrm{Z}$ & $\mathbf{P}$ \\
\hline \multirow{2}{*}{\begin{tabular}{|l|} 
Goal Setting/ \\
Mental Preparation
\end{tabular}} & Yes & 137 & 1,96 & 64 & \multirow{2}{*}{$-5,030$} & \multirow{2}{*}{$000^{*}$} \\
\hline & No & 265 & 1,58 & 69 & & \\
\hline \multirow{2}{*}{ Concentration } & Yes & 137 & 2,07 &, 58 & \multirow{2}{*}{$-4,666$} & \multirow{2}{*}{, $000^{*}$} \\
\hline & No & 265 & 1,75 & 64 & & \\
\hline \multirow{2}{*}{$\begin{array}{l}\text { Free } \\
\text { from Anxiety }\end{array}$} & Yes & 137 & 2,05 &, 80 & \multirow{2}{*}{$-4,042$} & \multirow{2}{*}{, $000^{*}$} \\
\hline & $\mathrm{No}$ & 265 & 1,74 & 78 & & \\
\hline \multirow{2}{*}{$\begin{array}{l}\text { High Performance } \\
\text { under Pressure }\end{array}$} & Yes & 137 & 1,33 & 82 & \multirow{2}{*}{$-2,149$} & \multirow{2}{*}{, $032^{*}$} \\
\hline & No & 265 & 1,13 &, 71 & & \\
\hline \multirow{2}{*}{\begin{tabular}{|l} 
Coping \\
with Difficulty
\end{tabular}} & Yes & 137 & 2,05 & ,70 & \multirow{2}{*}{$-5,357$} & \multirow{2}{*}{, $000^{*}$} \\
\hline & No & 265 & 1,64 &, 71 & & \\
\hline \multirow{2}{*}{$\begin{array}{l}\text { Confidence and } \\
\text { Achievement Motive }\end{array}$} & Yes & 137 & 2,32 & ,60 & \multirow{2}{*}{$-3,859$} & \multirow{2}{*}{, $000^{*}$} \\
\hline & No & 265 & 2,04 & 70 & & \\
\hline \multirow{2}{*}{$\begin{array}{l}\text { Coaching } \\
\text { Status }\end{array}$} & Yes & 137 & 2,32 & ,62 & \multirow{2}{*}{$-2,842$} & \multirow{2}{*}{, $004^{*}$} \\
\hline & No & 265 & 2,15 & 61 & & \\
\hline
\end{tabular}


According to Table 13, the difference was statistically significant in favor of the students, whose family has a licensed athlete, in all sub-dimensions of coping skills with sport problems depending on the variable of the licensed athlete in the family $(p<0.05)$.

Table 14: Analysis results of the research group by the sport branch variable

\begin{tabular}{|c|c|c|c|c|c|c|}
\hline Sub-dimensions & Sport Branch & $\mathbf{N}$ & $\bar{x}$ & SD & $\mathrm{Z}$ & $\mathbf{P}$ \\
\hline \multirow{2}{*}{$\begin{array}{l}\text { Goal Setting/ } \\
\text { Mental Preparation }\end{array}$} & Individual Sports & 168 & 1,63 & ,70 & \multirow{2}{*}{$-2,217$} & \multirow{2}{*}{, $027^{*}$} \\
\hline & Team Sports & 234 & 1,77 & 69 & & \\
\hline \multirow{2}{*}{ Concentration } & Individual Sports & 168 & 1,77 & 69 & \multirow{2}{*}{$-2,366$} & \multirow{2}{*}{, $018^{*}$} \\
\hline & Team Sports & 234 & 1,92 &, 59 & & \\
\hline \multirow{2}{*}{$\begin{array}{l}\text { Free from } \\
\text { Anxiety }\end{array}$} & Individual Sports & 168 & 1,76 & ,83 & \multirow{2}{*}{$-1,791$} & \multirow{2}{*}{,073 } \\
\hline & Team Sports & 234 & 1,91 & ,78 & & \\
\hline \multirow{2}{*}{$\begin{array}{l}\text { High Performance } \\
\text { under Pressure }\end{array}$} & Individual Sports & 168 & 1,18 & 74 & \multirow{2}{*}{,- 270} & \multirow{2}{*}{,787 } \\
\hline & Team Sports & 234 & 1,21 & 76 & & \\
\hline \multirow{2}{*}{$\begin{array}{l}\text { Coping } \\
\text { with Difficulty }\end{array}$} & Individual Sports & 168 & 1,71 & ,73 & \multirow{2}{*}{$-1,730$} & \multirow{2}{*}{084} \\
\hline & Team Sports & 234 & 1,83 & ,72 & & \\
\hline \multirow{2}{*}{$\begin{array}{l}\text { Confidence and } \\
\text { Achievement Motive }\end{array}$} & Individual Sports & 168 & 2,04 &, 67 & \multirow{2}{*}{$-2,633$} & \multirow{2}{*}{, $008^{*}$} \\
\hline & Team Sports & 234 & 2,21 & ,68 & & \\
\hline \multirow{2}{*}{$\begin{array}{l}\text { Coaching } \\
\text { Status }\end{array}$} & Individual Sports & 168 & 2,10 & 60 & \multirow{2}{*}{$-3,455$} & \multirow{2}{*}{, $001^{*}$} \\
\hline & Team Sports & 234 & 2,29 & 61 & & \\
\hline
\end{tabular}

Table 14 demonstrates a statistically significant difference in favor of the students doing team sports in the sub-dimensions of Goal setting / Mental preparation, Concentration, Confidence and Achievement motive and Being open to learning according to the sport branch variable of the research group $(\mathrm{p}<0.05)$.

Table 15: Analysis results of the research group by the variable of the number of trainings

\begin{tabular}{|c|c|c|c|c|c|}
\hline Sub-dimensions & Number of Trainings per Week & \begin{tabular}{|l|l|l|}
$\mathbf{N}$ & $\bar{x}$ & SD \\
\end{tabular} & $\mathbf{X}^{2}$ & $\mathrm{p}$ & Difference U Test \\
\hline \multirow{5}{*}{$\begin{array}{l}\text { Goal Setting/ } \\
\text { Mental Preparation }\end{array}$} & a. Once & \begin{tabular}{|l|l|l|}
67 & 1,14 & 54 \\
\end{tabular} & \multirow{5}{*}{106,708} & \multirow{5}{*}{, $000^{*}$} & \multirow{5}{*}{$\mathrm{a}, \mathrm{b}<\mathrm{c}, \mathrm{d}, \mathrm{e}$} \\
\hline & b. Twice & \begin{tabular}{|l|l|l|}
80 & 1,38 &, 55 \\
\end{tabular} & & & \\
\hline & c. 3 times & \begin{tabular}{|l|l|l|}
68 & $1,86,65$ \\
\end{tabular} & & & \\
\hline & d. 4 times & \begin{tabular}{|l|l|l|}
96 & 2,01 & 63 \\
\end{tabular} & & & \\
\hline & e. 5 times & $912,00,64$ & & & \\
\hline \multirow{5}{*}{ Concentration } & a. Once & \begin{tabular}{|l|l|l|}
67 & 1,36 &, 57 \\
\end{tabular} & \multirow{5}{*}{86,619} & \multirow{5}{*}{, $000^{*}$} & \multirow{5}{*}{$\mathrm{a}, \mathrm{b}<\mathrm{c}, \mathrm{d}, \mathrm{e}$} \\
\hline & b. Twice & \begin{tabular}{|l|l|l|}
80 & 1,57 &, 56 \\
\end{tabular} & & & \\
\hline & c. 3 times & \begin{tabular}{|l|l|l|}
68 & 2,05 &, 51 \\
\end{tabular} & & & \\
\hline & d. 4 times & \begin{tabular}{|l|l|l|}
96 & 2,14 &, 50 \\
\end{tabular} & & & \\
\hline & e. 5 times & \begin{tabular}{|l|l|l|}
91 & 2,03 & 66 \\
\end{tabular} & & & \\
\hline \multirow{5}{*}{$\begin{array}{l}\text { Free from } \\
\text { Anxiety }\end{array}$} & a. Once & $671,56,80$ & \multirow{5}{*}{28,085} & \multirow{5}{*}{, $000^{*}$} & \multirow{5}{*}{$\mathrm{a}, \mathrm{b}<\mathrm{c}, \mathrm{d}$} \\
\hline & b. Twice & \begin{tabular}{|l|l|l|}
80 & 1,66 &, 77 \\
\end{tabular} & & & \\
\hline & c. 3 times & \begin{tabular}{|l|l|l|}
68 & 1,98 &, 72 \\
\end{tabular} & & & \\
\hline & d. 4 times & \begin{tabular}{|l|l|l|}
96 & 2,13 &, 75 \\
\end{tabular} & & & \\
\hline & e. 5 times & \begin{tabular}{|l|l|l|}
91 & $1,82,85$ \\
\end{tabular} & & & \\
\hline \multirow{5}{*}{$\begin{array}{l}\text { High Performance } \\
\text { under Pressure }\end{array}$} & a. Once & \begin{tabular}{|l|l|l|}
67,83 &, 67 \\
\end{tabular} & \multirow{5}{*}{32,874} & \multirow{5}{*}{, $000^{*}$} & \multirow{5}{*}{$a<b, c, d, e$} \\
\hline & b. Twice & \begin{tabular}{|l|l|l|}
80 & 1,01 &, 57 \\
\end{tabular} & & & \\
\hline & c. 3 times & \begin{tabular}{|l|l|l|}
68 & 1,39 &, 76 \\
\end{tabular} & & & \\
\hline & d. 4 times & $961,20,70$ & & & \\
\hline & e. 5 times & \begin{tabular}{|l|l|l|}
91 & 1,48 & 84 \\
\end{tabular} & & & \\
\hline
\end{tabular}




\begin{tabular}{|c|c|c|c|c|c|}
\hline \multirow{5}{*}{$\begin{array}{l}\text { Coping } \\
\text { with Difficulty }\end{array}$} & a. Once & \begin{tabular}{|l|l|l|}
67 & $1,27,60$ \\
\end{tabular} & \multirow{5}{*}{67,215} & \multirow{5}{*}{, $000^{*}$} & \multirow{5}{*}{$\begin{array}{l}\mathrm{a}<\mathrm{b}, \mathrm{c}, \mathrm{e} \\
\mathrm{b}<\mathrm{c}, \mathrm{d}, \mathrm{e}\end{array}$} \\
\hline & b. Twice & \begin{tabular}{l|l|l|}
80 & $1,52,66$ \\
\end{tabular} & & & \\
\hline & c. 3 times & \begin{tabular}{|l|l|l|}
68 & 1,93 \\
\end{tabular} & & & \\
\hline & d. 4 times & $962,08,71$ & & & \\
\hline & e. 5 times & $91 \mid 1,97,71$ & & & \\
\hline \multirow{5}{*}{$\begin{array}{l}\text { Confidence and } \\
\text { Achievement Motive }\end{array}$} & a. Once & \begin{tabular}{|l|l|}
67 & $1,62,74$ \\
\end{tabular} & \multirow{5}{*}{74,075} & \multirow{5}{*}{, $000^{*}$} & \multirow{5}{*}{$\mathrm{a}, \mathrm{b}<\mathrm{c}, \mathrm{d}, \mathrm{e}$} \\
\hline & b. Twice & $80 \mid 1,87,63$ & & & \\
\hline & c. 3 times & $68 \mid 2,27,57$ & & & \\
\hline & d. 4 times & $962,42,54$ & & & \\
\hline & e. 5 times & $91 \mid 2,36,62$ & & & \\
\hline \multirow{5}{*}{$\begin{array}{l}\text { Coaching } \\
\text { Status }\end{array}$} & a. Once & \begin{tabular}{|c|c|c|}
67 & $1,88,63$ \\
\end{tabular} & \multirow{5}{*}{55,899} & \multirow{5}{*}{, $000^{*}$} & \multirow{5}{*}{$\mathrm{a}, \mathrm{b}<\mathrm{c}, \mathrm{d}, \mathrm{e}$} \\
\hline & b. Twice & $80 \mid 1,95,60$ & & & \\
\hline & c. 3 times & \begin{tabular}{|l|l|l|}
$68,38,54$ \\
\end{tabular} & & & \\
\hline & d. 4 times & $962,45,54$ & & & \\
\hline & e. 5 times & $912,31,57$ & & & \\
\hline
\end{tabular}

Table 15 shows a statistically significant difference in favor of the students who train three times or more per week in all the sub-dimensions of coping skills with sport problems depending on the variable of number of trainings per week $(p<0.05)$.

Table 16: Analysis results of the research group by the self-definition variable

\begin{tabular}{|c|c|c|c|c|c|c|}
\hline Sub-dimensions & Self-Definition & $\mathbf{N}$ & $\bar{x}$ & SD & $\mathrm{Z}$ & $\mathbf{P}$ \\
\hline \multirow{2}{*}{\begin{tabular}{|l|} 
Goal Setting/ \\
Mental Preparation
\end{tabular}} & Asocial-Introvert & 183 & 1,55 & 68 & \multirow{2}{*}{$-4,356$} & \multirow{2}{*}{, $000^{*}$} \\
\hline & Social-Extrovert & 219 & 1,85 & 68 & & \\
\hline \multirow{2}{*}{ Concentration } & Asocial-Introvert & 183 & 1,71 & 63 & \multirow{2}{*}{$-4,433$} & \multirow{2}{*}{, $000^{*}$} \\
\hline & Social-Extrovert & 219 & 1,98 &, 62 & & \\
\hline \multirow{2}{*}{\begin{tabular}{|l} 
Free from \\
Anxiety
\end{tabular}} & Asocial-Introvert & 183 & 1,70 & 84 & \multirow{2}{*}{$-3,121$} & \multirow{2}{*}{, $002^{*}$} \\
\hline & Social-Extrovert & 219 & 1,97 & 75 & & \\
\hline \multirow{2}{*}{$\begin{array}{l}\text { High Performance } \\
\text { under Pressure }\end{array}$} & Asocial-Introvert & 183 & 1,11 & ,72 & \multirow{2}{*}{$-1,767$} & \multirow{2}{*}{,077 } \\
\hline & Social-Extrovert & 219 & 1,26 & ,77 & & \\
\hline \multirow{2}{*}{ Coping with Difficulty } & Asocial-Introvert & 183 & 1,60 & 74 & \multirow{2}{*}{$-4,521$} & \multirow{2}{*}{, $000^{*}$} \\
\hline & Social-Extrovert & 219 & 1,93 & 69 & & \\
\hline \multirow{2}{*}{$\begin{array}{l}\text { Confidence and } \\
\text { Achievement Motive }\end{array}$} & Asocial-Introvert & 183 & 2,01 & ,68 & \multirow{2}{*}{$-3,714$} & \multirow{2}{*}{, $000^{*}$} \\
\hline & Social-Extrovert & 219 & 2,25 &, 67 & & \\
\hline \multirow{2}{*}{$\begin{array}{l}\text { Coaching } \\
\text { Status }\end{array}$} & Asocial-Introvert & 183 & 2,12 & 63 & \multirow{2}{*}{$-2,772$} & \multirow{2}{*}{, $006^{*}$} \\
\hline & Social-Extrovert & 219 & 2,29 &, 59 & & \\
\hline
\end{tabular}

Table 16 presents a statistically significant difference in favor of those who define themselves social-extroverted in all of the sub-dimensions of coping skills with sport problems except for high performance under pressure according to the self-definition variable of the research group $(p<0.05)$.

\section{Discussion}

In our study, it was determined that there was a statistically significant difference in favor of male students in terms of goal setting / mental preparation, concentration, high 
performance under pressure and coping with difficulty according to the gender variable. The study of Elferink-Gemser, Visscher and Lemmink (2005) put forward that boys had higher scores than girls (among athletes with an age average of 14.8) in factors of selfconfidence, anxiety control, mental preparation and imagination factors in terms of their sports psychological abilities. In another study in which the relationship between continuous sportive self-confidence and gender variable was examined, it was determined that male participants had a higher level of continuous sporting selfconfidence compared to females. It is stated that this might result from the fact that males have a higher self-confidence in terms of motoric features and general sporting confidence than females (Corbin, 1981; Vurgun, 2010). In general, it is remarked that new longitudinal studies are needed for gender specific development of psychological skills in sports during adolescence; and it should be considered that, in terms of psychological change, boys and girls might develop differently in the background of this period because of the rapid physical change (Christie, 2005).

According to the High School type variable of the research group, it was determined that there was a statistically significant difference in all sub-dimensions excluding the sub-dimension of coaching status. Taking into account the source of the difference, the fact that the difference is in favor of the students in the sports high schools suggests that they are more capable in overcoming sporting problems due to their experience and involvement in more sporting activities than other high schools.

In accordance with the self-definition variable of the research group, it was found that there was a statistically significant difference in favor of those who defined themselves social-extroverted in all sub-dimensions of coping skills with sport problems except for high performance under pressure. In many similar studies, it was also found that the extroversion characteristic of the participants doing sports was better (Egloff and Gruhn, 1996; Courneya and Hellsten, 1998; Blanco et al., 1999).

It was determined that there was a statistically significant difference only in the goal setting / mental preparation sub-dimension according to the education level of the parents.

It was specified that was a statistically significant difference only in coping with difficulty sub-dimension of the research group according to the play type liked.

A statistically significant difference was observed in the goal setting / mental preparation, concentration, high performance under pressure and coping with difficulty sub-dimensions according to the sport purpose variable of the research group.

It was designated that there was a statistically significant difference in the subdimensions of goal setting / mental preparation, concentration, confidence and achievement motive and being open to learning in favor of the students doing team sport according to the variable of sport branch of the research group. With respect to the results of the research aimed to compare the self-efficacy levels of individuals who were between 9-13 years and did or did not do sports; it was ascertained that it was parallel to our study in terms of sportive psychological abilities, there was a statistically significant difference in the self-efficacy scores of students who did team sport and did not do sports, and the self-efficacy scores of female students in the team sport group were higher than male 
students (Öztürk and Şahin, 2007). Vanek's and Caratty's book "Psychology and the Superior Athlete" (1970), which support our study and reached similar results, concluded that team athletes were less anxious than the individual athletes and their selfconfidence was higher. The reasons such as that the athletes competing in team sports have a high self-confidence and get support from their team mates while struggling against difficulties, the negativities that may occur during and after the competition concern the whole team and the responsibility is shared suggest that they are better than individual athletes in the fight against stress.

Depending on the sport year variable of the research group, it was seen that the difference was in the group who had been doing sports for 6-7 years and 8 years and more in all of the sub-dimensions of coping with sport problems. Therefore, it is seen that the increase in the sport year affects positively the sports-specific psychological ability level. Coşkun's study (2018), which supports our findings, demonstrates that as the age of sports increases, the sports psychological skill levels of the participants increase, and there are statistically significant differences in favor of those with higher sport year among the groups in terms of all factors and general levels of psychological skills (Coşkun, 2018).

According to the variables of being club licensed and playing in school teams, it was determined that the difference was in favor of those who actively participated in these activities in all sub-dimensions of sport-specific psychological ability levels. This situation shows that there is a significant difference between the sports-specific psychological ability levels of sportive and sedentary students. Based on available findings, we can say that sportive students act in a planned way to achieve the goal, can concentrate without being influenced by external factors while performing sports and can show better performance under high pressure, display the best performance in the face of difficult situations by keeping the situation under control, can always be positively motivated by their self-confidence and are open to learning and improving themselves by following the directives given.

Depending on the variable of the number of weekly trainings, it was determined that the difference was statistically significant in favor of the students exercising three times or more per week in all of the sub-dimensions of coping with sporting problems. Examining the results of the study investigating the psychological effects of exercise frequency, it was reported that those with a high frequency of exercise had lower levels of loneliness points than those with low frequency (Yaziclar, 2004, Page RM, Tucker LA, 1994, Hopman RM, Westhoff MH, 2002). In the study conducted by Canan and Ataoğlu in 2010, it was found that depression and anxiety symptoms decreased as the time spent doing sports increased, and there was a linear relationship between this time and perception of problem solving skill (Canan and Ataoğlu, 2010). The results obtained in our study are in line with the literature.

On the basis of the variable of licensed athletes in the family of the research group, it was found that the difference was statistically significant in favor of the students with licensed athletes among the family members in all sub-dimensions of coping skills with sport problems. 
As a result of our study, it has been seen that the students who are successful in bilateral relations, social, have athletic identities in the forefront and are interested in sports for a long time are more advanced in terms of sports-specific psychological abilities and goal setting, concentration, being free from anxiety, high performance under pressure, coping with difficulties, confidence and achievement motive and being open to learning. Thus, sports environments can provide positive contributions to the development of sports-specific psychological skills by providing students with such appropriate areas.

As a result, it was asserted that psychological factors were more effective in determining performance compared to physical evaluations (Smith and Christensen, 1995). Today, the concept of psychological skills has become a very important phenomenon in the world of sports, and the scientists who steer the world of sports have gone into a great effort to elevate the performance to the highest levels. They develop new training models to maximize the efficiency of the athlete and explore the ways to improve their performance, and psychological skills are seen as the combination of motivating elements that maximize the improved performance. However, although the concept of psychological skill is one of the most important methods that provide sporting efficiency in the world, it is obvious that it is not properly used in our country. Therefore, increasing the number of the studies on psychological skills in our country will make positive contributions to the literature.

\section{Conclusion}

In the light of the results obtained from our study;

1) When the data obtained from our study and the studies conducted are examined, it was seen that male athletes used strategies related to sport-specific psychological abilities more than female athletes (Elferink-Gemser, Visscher and Lemmink (2005), Corbin, 1981). At this point, coaches and sports psychologists can work extra with female athletes to support them in developing their sports-specific psychological abilities and help them improve their sporting performance.

2) Coaches and trainers prepare the necessary environment to cope with the sportsspecific psychological factors; and sports psychologists can provide support and managers can be developed in this regard.

3) The number of the studies based on personality traits of students can be increased in terms of contributing to the development of sports-specific psychological ability.

4) From the viewpoint of athletes, athletes should be willing to train themselves and be open to all kinds of support in developing their sports-specific psychological abilities.

5) In the period of beginning sports, the basic psychological views of the athletes should be revealed, strategies to develop the sports-specific psychological skills should be taught to athletes from a certain age, and in the design of training, the practices of working conditions appropriate to the competition conditions should 
be prioritized and entertaining activities in the play format should be included. Since the sporting activity started at a young age in a play format will enable the athlete to do sports in a healthier way physically, mentally and psychologically, for maximum performance, attention should be paid to keep athletes away from training and competitions where maximum success is expected from early ages.

\title{
Conflict of Interest
}

No potential conflict of interest was reported by the authors.

\begin{abstract}
About the Authors
Doctor Lecturer Kemal Kurak, Çanakkale Onsekiz Mart University, Faculty of Sport Sciences, Department of Recreation, Çanakkale, Turkey. Research interests: sport science, movement and training, coaching, football, sport psychology.

Research Assistant Hakan Büyükçelebi, İnönü University, Faculty of Sport Sciences, Department of Coaching Education, Malatya, Turkey. Research interests: sport science and technology, movement and training, analysis in sport, football analysis, sport psychology.
\end{abstract}

\section{References}

Akarçeşme C, 2004. The Relationship Between Pre-Competition State Anxiety and Performance Criteria in Volleyball, Master Thesis, Gazi University Institute of Health Sciences, Ankara.

Aktaş S. 2009. The Effect of Balance on Performance of Elite Alpine Skiers. Niğde University Institute of Social Sciences, PhD Thesis, Nigde University.

Bakırcıoğlu R. 2012. Encyclopedic Dictionary of Education and Psychology, Ankara, Turkey.

Blanco S, Hill CD, Piedmont RL, 1999. Predicting Athletic Performance Using the FiveFactor Model of Personality. Personality and Individual Differences 27: 769-777.

Canan F, Ataoğlu A, 2010. The influence of sports on anxiety, depression, and perceived problem solving ability. Anatolian Journal of Psychiatry, 11: 38-43.

Cevizci A, 1999. Philosophical Dictionary, İstanbul, Turkey.

Christie D, 2005. Adolescent development. British Medical Journal, 330(7486): 301-304.

Corbin CB, 1981. Sex of subject, sex of opponent, and opponent ability as factors affecting self-confidence in a competitive situation. Journal of Sport Psychology, 3: 265-270.

Courneya KS, Hellsten LAM, 1998. Personality Correlates of Exercise Behavior, Movives, Barriers and Preferences: An Application of the Five-Factor Model. Personality and Individual Differences, 24(5): 625-633.

Coşkun K, 2018. Psychological Skill Levels of Amateur and Professional Football Players. Master Thesis. Erzincan University Institute of Health Sciences, Erzincan. 
Çetinkaya T, 2015. Athlete identity and continuous sportive self-confidence relationship in team sports. PhD Thesis, Gazi University Institute of Education Sciences, Ankara.

Efe M, Öztürk F, Koparan Ş, Şenışık Y, 2008. The Effect of Volleyball Training on Social Efficacy Expectation and Assertiveness at 14-16 Age Group Individuals. Journal of Uludağ University Education Faculty, 20(1): 69-97.

Elferink-Gemser MT, 2005. Today's talented youth field hockey players, the stars of tomorrow, PhD Thesis.

Egloff B, Gruhn AJ, 1996. Personality and Endurance Sports. Personality and Individual Differences, 21(2): 223-229.

Gee CJ, Marshall JC, King JF, 2010. Should coaches use personality assessments in the talent identification process? A 15 year predictive study on professional hockey players. International Journal of Coaching Science, 4: 25-34.

Hagger M, Chatzisarantis N, 2005. The Social Psychology of Exercise and Sport, New York, USA.

Harwood C, Cumming J, Fletcher D, 2004. Motivational profiles and psychological skills use within elite youth sport. Journal of Applied Sport Psychology, 16: 318-332.

Hayslip B, Petrie TA, MacIntire MM, Jones GM, 2010. The influences of skill level, anxiety, and psychological skills use on amateur golfers' performances. Journal of Applied Sport Psychology, 22: 123-133.

Hopman RM, Westhoff MH, 2002. Health education and exercise stimulation for older people: development and evaluation of the program -Healty and Vital-, Tijdschrift voor Gerontologie en Geriatrie, 33(2): 56-63.

Koç H, 2004. Evaluation of the factors affecting the state anxiety levels of professional football players, Master Thesis, Dumlupinar University Institute of Social Sciences, Kütahya.

Lazarus RS, Folkman S, 1984. Stress, Appraisal and Coping, New York, USA.

Malina RM, Ribeiro B, Aroso J, Cumming SP, 2007. 'Characteristics of youth Soccer Players Aged 13-15 Years Classified by Skill Level', British Journal of Sports Medicine, 41: 290-295.

McCarthy PJ, Jones MV, Harwood CG, Olivier S, 2010. What do young athletes implicitly understand about psychological skills? Journal of Clinical Sport Psychology, 4(2): 158-172.

Özcan V, Günay M, 2017. The Turkish Adaptation of Athletic Coping Skills Inventory-28 (ACSI-28): The validity and reliability study. Turkish Journal of Sport and Exercise, 19(1):130-136.

Öztürk F, Şahin Ş, 2007. Comparison of the social self efficacy results of 9-13 age groups individuals who do sports and don't do (Bursa sample). İlköğretim Online, 6(3): 469-479.

Page RM, Tucker LA, 1994. Psychosocial discomfort and exercise frequency: epidemiological study of adolescents, adolescence. National Library of Medicine, 29(113):183-91. 
Smith RE, Christensen, DS, 1995. Psychology skills as predictors of performance and survival in professional baseball. Journal of Sport and Exercise Psychology, 17: 399-415.

Smith RE, Schutz RW, Smoll FL, Ptacek JT, 1995. Development and validation of a multidimensional measure of sport-specific psychological skills: The athletic coping skills inventory-28. Journal of Sport \& Exercise Psychology, 17: 379-389

Tavacıoğlu L, 1999. Sports Psychology, Ankara, Turkey.

Vanek M, Cratty BJ, 1970. Psychology and the Superior Athlete, London, England.

Vurgun T, 2010. Adapting the sports imagery questionnaire to Turkish and the effect of imagination in sports on competition anxiety and sportive confidence. PhD Thesis, Ege University Institute of Health Sciences.

Weinberg R, Miller A, Horn T, 2012. The influence of a self-talk intervention on collegiate cross-country runners. International Journal of Sport and Exercise Psychology, 10(2): 123-134.

Yazıcilar I, 2004. Investigating the loneliness levels of young people who do sports and those who do not. Master Thesis, Celal Bayar University. 
Kemal Kurak, Hakan Büyükçelebi

INVESTIGATION OF SPORTS-SPECIFIC PSYCHOLOGICAL ABILITY LEVELS OF HIGH SCHOOL STUDENTS

Creative Commons licensing terms

Authors will retain the copyright of their published articles agreeing that a Creative Commons Attribution 4.0 International License (CC BY 4.0) terms will be applied to their work. Under the terms of this license, no permission is required from the author(s) or publisher for members of the community to copy, distribute, transmit or adapt the article content, providing a proper, prominent and unambiguous attribution to the authors in a manner that makes clear that the materials are being reused under permission of a Creative Commons License. Views, opinions and conclusions expressed in this research article are views, opinions and conclusions of the author(s). Open Access Publishing Group and European Journal of Physical Education and Sport Science shall not be responsible or answerable for any loss, damage or liability caused in relation to/arising out of conflict of interests, copyright violations and inappropriate or inaccurate use of any kind content related or integrated on the research work. All the published works are meeting the Open Access Publishing requirements and can be freely accessed, shared, modified, distributed and used in educational, commercial and non-commercial purposes under a Creative Commons attribution 4.0 International License (CC BY 4.0). 